ARTIGO

Recebido em: 27/04/2016

Aceito em:

$10 / 11 / 2016$

\title{
Expressão científica de Mato Grosso na pesquisa brasileira sobre indicadores socioambientais no agronegócio na base de dados Cab Abstracts
}

\author{
Scientific expression of Mato Grosso in Brazil's research on social \\ and environmental indicators in Agribusiness in Cab Abstracts \\ database
}

\footnotetext{
Maykon Jesse Barbieri de CAMARGo (maykon_adm@hotmail.com)* - Dejânia Vieira de

ARAÚJO (dejania@unemat.br)** - Rogério MUGNAINI (mugnaini@usp.br)***

* Mestre em Meio Ambiente e Sistema de Produção Agrícola pela Universidade do Estado de Mato Grosso e Administrador da Rural Soluções e Serviços Ltda.

** Professora da Universidade do Estado de Mato Grosso do Departamento de Agronomia, Campus de Tangará da Serra/MT; do Programa de Pós-graduação em Ambiente e Sistemas de Produção - PPGASP e do Programa de Pós-graduação em Genética e Melhoramento de Plantas - PGMP.

*** Professor da Universidade de São Paulo, na Escola do Comunicação e Artes e do Programa de Pósgraduação em Ciência da Informação - PPGCI.
}

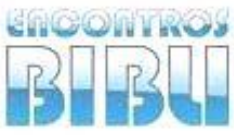

v. 22, n. 48,2017

p. 73-87

ISSN 1518-2924

\section{Resumo}

O objetivo deste trabalho foi de avaliar a expressão científica de Mato Grosso na pesquisa brasileira sobre indicadores socioambientais no agronegócio por meio dos indicadores bibliométricos, utilizou-se como fonte os artigos de periódicos indexados na base de dados Cab Abstracts entre 2005 e 2014. Os principais resultados encontrados foram a evolução por ano; a distribuição no Brasil em termos de regiões, estados e municípios; a produção dos autores que mais publicaram; as instituições publicadoras dos trabalhos; e os periódicos mais utilizados. Com os resultados foi possível verificar que o tema estudado vem ganhando espaço na comunidade científica e representatividade nos últimos anos, situação que não ocorre com o Estado de Mato Grosso, pois este não possui trabalhos indexados na base de dados estudada.

Palavras-chave: Ciência da Informação. Meio Ambiente. Agricultura. Bibliometria.

\begin{abstract}
The objective of this study was to evaluate the scientific expression of Mato Grosso in the Brazilian research on socio-environmental indicators in agribusiness through bibliometric indicators, we used as a source, some papers from indexed journals in the database of Cab Abstracts between 2005 and 2014 The main results we found were the evolution per year; distribution in Brazil in terms of regions, states and municipalities; the production of authors who published the most; the publishing institutions of the paper; and the most used journals. We could verify with the results that the issue studied has been gaining ground in the scientific community and representability in recent years, a situation that does not occur with the state of Mato Grosso, since it does not have indexed papers in the studied database. Keywords: Information Science. Environment. Agriculture. Bibliometrics.
\end{abstract}




\section{INTRODUÇÃO}

A produção do conhecimento científico é produto do trabalho de pesquisadores, que têm como uma das suas principais responsabilidades a publicação dos resultados de pesquisa em periódicos e eventos para a comunidade científica da qual fazem parte (MACHADO JÚNIOR, 2013).

A comunicação científica desempenha função de suma importância porque consiste na divulgação dos resultados das pesquisas à comunidade científica e a outros especialistas interessados, de forma a favorecer a geração e a disseminação de conhecimentos e de atividades de pesquisas. É a troca de informações científicas que favorece ao produto (produção científica) e aos produtores (pesquisadores) a necessária visibilidade e possível credibilidade no meio social em que os mesmos se inserem (MACHADO JÚNIOR, 2013).

A bibliometria consiste na medida quantitativa das publicações científicas de um pesquisador ou instituição, em periódicos com seleção arbitrada, e a medida qualitativa destas publicações por meio de indicadores que incluem estudos comparativos de publicações e citações (PRITCHARD, 1998 apud SOUZA, 2011). Os indicadores bibliométricos podem ser compreendidos como dados estatísticos usados para medir algo intangível. A construção e o uso de indicadores de produção científica são objeto de estudo de várias áreas do conhecimento, utilizadas para o planejamento e a execução de políticas para o setor e também para que a comunidade científica conheça melhor o sistema no qual está inserida (SOUZA, FARIA, 2013).

A bibliometria vem se desenvolvendo nos últimos tempos, e esse processo contínuo de transformar dados brutos em conhecimento estratégico, acompanha esse crescimento, e como consequência, a importância e o valor dos indicadores se pautam na possibilidade de quantificar algo intangível, como a geração de conhecimentos, e esses indicadores de produção científica devem ser elaborados voltados ao avanço da ciência e da tecnologia e o progresso econômico e social.

Os indicadores de atividade científica têm sido utilizados nos últimos anos como importante ferramenta na gestão de política científica e foram vislumbrados na atual pesquisa como ferramenta capaz de permitir averiguar o monitoramento e a visibilidade da produtividade frente a Cab Abstracts, tendo como tema os Indicadores Socioambientais no Agronegócio.

Os trabalhos bibliométricos da produção científica indexados a uma base de dados constituem-se instrumentos adequados de gestão bibliográfica, portanto este estudo teve como objetivo avaliar a expressão científica de Mato Grosso na pesquisa brasileira sobre indicadores socioambientais no agronegócio na base de dados internacional Cab Abstracts a partir da quantidade de artigos indexados entre os anos de 2005 a 2014.

Por meio do descrito, avalia-se como oportuna a tentativa de apresentar as principais características do desenvolvimento da ciência brasileira, com enfoque no ponto de vista regional, e entre os pesquisadores, pois acredita-se que isso permita colaborar para o melhor entendimento do funcionamento e evolução do sistema de produção científica.

\section{REFERENCIAL TEÓRICO}

A construção de indicadores tem sido a ferramenta mais utilizada por aqueles que objetivam mensurar o estágio de desenvolvimento de países, regiões, locais e comunidades, embora muitas vezes se obtenham apenas índices de crescimento, por meio de variáveis econômicas (HENRIQUEZ et al, 2013).

O ambiente complexo, incerto e instável de hoje (e a problemática socioambiental, por exemplo) exige abordagens sistêmicas e integradas, capazes de indicar caminhos alternativos para os novos e antigos desafios que se apresentam (HENRIQUEZ et al, 2013).

Por meio do processo maçante de degradação e deterioração do meio ambiente ocorrido nas últimas décadas, faz-se necessário o aumento da preocupação em se criar indicadores que incluam uma visão sistêmica, tendo em vista que esses novos pensamentos devem partir prioritariamente por quem trabalha com a agricultura, destacando-se o agronegócio.

No Brasil, o agronegócio é um segmento importante economicamente por gerar emprego e renda e se destacar no comércio mundial, conforme afirmam Assad et al. (2012), o Brasil é o terceiro maior exportador mundial de produtos agrícolas e lidera o ranking de 
exportação de produtos como o açúcar, a carne de frango, a carne bovina, o café, o suco de laranja, o tabaco e o álcool.

Quando se trata do estado de Mato Grosso, o agronegócio é a base da economia, a agropecuária representa quase $30 \%$ do Produto Interno Bruto estadual, e se tornou um dos estados brasileiros de maior presença no mercado internacional com exportação de commodities, principalmente grãos (SEPLAN, 2011).

\section{MATERIAL E MÉTODOS}

Para a execução desta pesquisa, a metodologia utilizada foi embasada nos métodos dos trabalhos de Puerta (2012) e Andrade (2012) e está estruturada no conjunto de etapas planejadas, sendo de caráter exploratório com abordagem quantitativa.

Para a estruturação e elaboração dos indicadores bibliométricos, o trabalho baseouse em:

a) Levantamento da literatura em indicadores socioambientais, agronegócio e bibliometria;

b) Análise das bases de dados disponíveis e definição da mais adequada;

c) Identificação da lista de termos;

d) Formulação da expressão de busca para indicadores socioambientais e agronegócio;

e) Coleta de dados bibliográficos da produção científica em ambas as buscas na base de dados;

f) Análise dos dados com uso de software para tratamento bibliométrico, geração de dados quantitativos e identificação de indicadores bibliométricos.

Quanto a definição da base de dados, houve a escolha intencional da Cab Abstracts pois acredita-se ser uma das mais representativas para o efeito da pesquisa. A Cab (Commonwealth Agricultural Bureau) Abstracts, de acordo com Puerta (2012) é a base de dados bibliográfica que representa a fonte mais abrangente de informações de pesquisa internacional nos campos de agricultura e ciências da vida, produzida pela CABI Publisching.

Findada a etapa de análise e seleção da base de dados, a sequência do trabalho foi a definição da estratégia e das expressões de busca para recuperar os dados bibliográficos da produção científica brasileira.

Para a definição das expressões de buscas foram identificadas palavras-chaves representativas dos conteúdos sobre indicadores socioambientais e agronegócio, nos artigos científicos, teses, dissertações, análise de periódicos, trabalhos acadêmicos e sites da internet, previamente selecionados em uma pesquisa bibliográfica.

A partir das expressões de busca foram definidas a estratégia de busca, a primeira para Indicadores Socioambientais e a segunda para Agronegócio, com uso de símbolos de truncagem $\$ \mathrm{e}^{*}$ e os operadores booleanos $O R$ e $A N D$.

A decisão pela delimitação do período de busca compreendeu os anos de 2005 a 2014, enquanto que a abrangência geográfica foi delimitada para o Brasil, incluindo-se produções brasileiras publicadas tanto no Brasil quanto no exterior.

Em virtude do trabalho versar sobre dois temas, as estratégias de buscas foram aplicadas separadamente para cada uma das temáticas e, com os resultados da recuperação dos dois assuntos na base de dados, foi feita a junção de ambos para se obter o corpus da pesquisa.

A estratégia de busca teve o intuito de abranger de maneira ampla os artigos publicados em revistas científicas relacionados aos indicadores socioambientais, e teve como principais fontes para composição da lista de termos as pesquisas de Louette (2009) e Gamba (2011), a partir delas chegou-se aos dados constantes nas etapas 1 a 4 da Tabela 1, os quais relacionam uma lista de termos, indicadores e índices atrelados ao tema.

Na etapa cinco da Tabela 1 foi efetuado a relação dos resultados das quatro primeiras etapas com o uso do operador booleano $O R$, e posteriormente, buscou-se atrelar esse resultado com o termo Brasil, para que se buscasse as produções brasileiras, resultando na etapa 6.

No que se referiu ao termo Agronegócio, foi utilizada a estratégia definida por Puerta (2012) no trabalho que teve a colaboração de alguns especialistas da área da Empresa Brasileira de Pesquisa Agropecuária (Embrapa), do Instituto de Economia Agrícola e do 
Instituto de Pesquisas Tecnológicas (IPT), que trataram diretamente a temática do agronegócio, conforme é evidenciado na etapa 7 da Tabela 1.

Após isso, da mesma forma que foi feita com o termo de Indicadores Socioambientais, buscou-se atrelar o resultado dos termos de Agronegócio com o termo Brasil, para que se buscasse as produções brasileiras, que gerou a etapa 8 .

Com os resultados dos dois temas, a finalização da estratégia de busca foi a relação deles para se encontrar as publicações que trataram de ambos, especificado nas etapas 9 e 10 da Tabela 1.

0 resumo e a representação de toda a estratégia e das expressões de buscas utilizadas na pesquisa estão apresentadas na Tabela 1:

Tabela 1: Estratégia de busca sobre indicadores socioambientais no agronegócio na base Cab Abstracts, 2005-2014.

\begin{tabular}{|c|c|}
\hline Etapas & Termo de Expressão de Busca \\
\hline$\# 1$ & $\begin{array}{l}\text { TS=(environmental indicator\$ OR environmental indicator\$ partner OR socio-environmental indicator\$ OR social } \\
\text { indicator\$ OR sustainable development OR indicator\$ of environmental sustainability OR sustainable development } \\
\text { indicator\$ OR environmental indicator system\$ OR risk indicator\$ and environmental vulnerability) }\end{array}$ \\
\hline$\# 2$ & $\begin{array}{l}\text { TS=(measure of economic welfare OR mew OR genuine progress indicator OR gpi OR adjusted net savings OR } \\
\text { genuine saving indicator OR gsi OR genuine investment OR gpd-green OR global environment outlook OR geo OR } \\
\text { global environment outlook geo OR geo brazil OR municipal social indicator\$) }\end{array}$ \\
\hline$\# 3$ & $\begin{array}{l}\text { TS=(index of sustainable economic welfare OR isew OR human development index OR hdi OR human poverty index } \\
\text { OR hpi OR development index gender OR extent of participation by gender OR environmental degradation index } \\
\text { OR compass of sustainability OR living planet index OR pli OR index living planet OR ecological footprint OR ef RO } \\
\text { environmental sustainability index OR esi OR environmental performance index OR epi OR environmental } \\
\text { vulnerability index OR evi OR barometer of sustainability OR dashboard of sustainability OR well-being of nations } \\
\text { OR human well-being index OR hwi OR ecosystem well-being index OR ewi OR dna index - brazil OR index dna } \\
\text { brazil OR multiple indicator\$ system OR sustainable development indicator\$ - ibge OR geo world OR geo américa } \\
\text { latina e caribe OR geo cit\$ OR gross national happiness OR gnh OR barometer of inequalit\$ and poverty OR } \\
\text { accounting balance of nations OR quality indicator } \$ \text { Calvert-Henderson life OR happy planet index OR hpi OR index } \\
\text { of economic well-being OR iewb OR são paulo index of social responsibility OR index social health OR ish OR } \\
\text { responsible competitiveness index OR rci OR social footprint OR social development index OR quality score of } \\
\text { municipalities OR socioeconomic development index) }\end{array}$ \\
\hline$\# 4$ & TS=(agenda 21 OR bellagio principles OR the limits to growth) \\
\hline$\# 5$ & $\# 1$ OR \#2 OR \#3 OR \#4 \\
\hline \#6 & \#5 AND CU=BRAZIL \\
\hline$\# 7$ & $\begin{array}{l}\text { TS=(agricult* OR agribusiness OR agrobusiness OR agroindustr* OR farming OR "crop production" OR tillage OR } \\
\text { "animal husbandry" OR livestock OR agrochemic* OR pesticide* OR fertilizer* OR agri-food* OR agrifood* OR } \\
\text { herbicide*) }\end{array}$ \\
\hline$\# 8$ & \#7 AND CU=BRAZIL \\
\hline$\# 9$ & \#5 AND \#7 \\
\hline$\# 10$ & \#9 AND CU=BRAZIL \\
\hline
\end{tabular}

Fonte: dados do autor (2015).

As buscas na base de dados foram realizadas em estágio de pesquisa no Departamento de Biblioteconomia e Documentação da Escola de Comunicação e Artes da Universidade de São Paulo, entre os dias 13 a 17 de julho de 2015.

0 uso das estratégias de busca de maneira combinada proporcionou a recuperação de 746 registros bibliográficos no período de 2005 a 2014, retiradas 76 duplicações, restaram 670 que compuseram o corpus da pesquisa.

O tratamento bibliométrico foi elaborado com auxílio dos softwares My Endnote Web e Microsoft Excel, que proporcionaram análise individual dos campos da base de dados.

A elaboração dos indicadores bibliométricos proporcionou retratar a produção brasileira publicada no Brasil e no exterior e indexadas na base Cab Abstracts; a evolução das publicações por ano; a distribuição das pesquisas no Brasil em termos de regiões, estados e municípios; a produtividade dos autores; as instituições de publicação dos trabalhos; e os periódicos mais utilizados para publicação, com a classificação Qualis nas áreas interdisciplinar e ciências agrárias. 


\section{RESULTADOS E DISCUSSÃO}

Neste item são apresentadas as análises da evolução anual da produção científica sobre indicadores socioambientais no agronegócio, publicados entre 2005 a 2014, indexados na Cab Abstracts, o que resultou em 670 registros bibliográficos recuperados.

A quantidade de registros nacionais distribuídos por cada ano na base de dados está apresentada na Figura 1, que esclarece como foi a distribuição dos artigos no decorrer dos dez anos da pesquisa, compreendendo abordagem dos dados gerais, das publicações feitas no exterior e as divulgadas em revistas brasileiras.

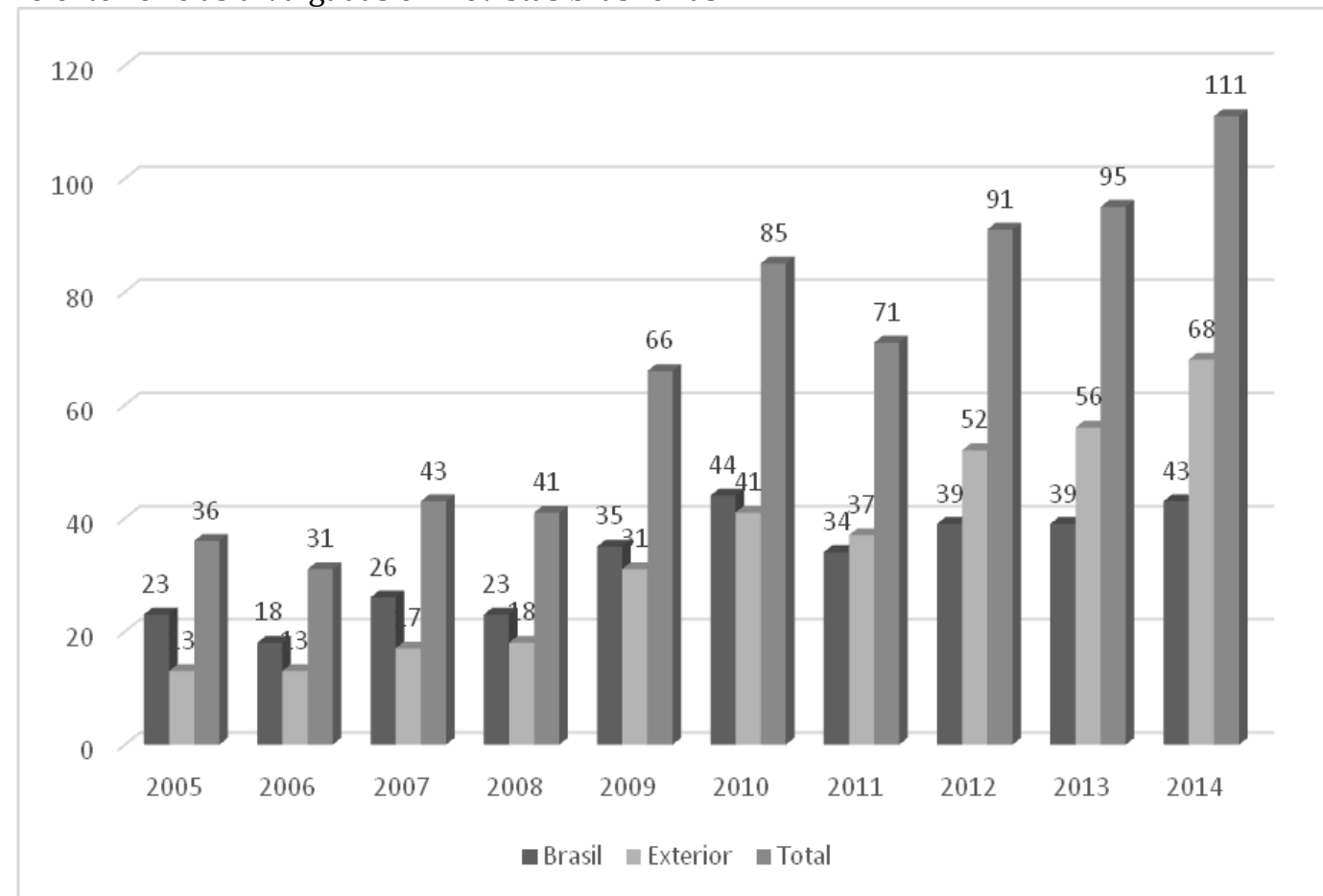

Figura 1: Distribuição anual das publicações brasileiras sobre indicadores socioambientais no agronegócio na base Cab Abstracts, 2005-2014.

Fonte: dados do autor a partir da pesquisa na Cab Abstracts (2015).

A produtividade apresentada na Figura 1 não se mantém constante no período pesquisado, pois existem alguns anos em que a quantidade de publicações cai em comparação com o período anterior, como nos casos dos anos de 2005 para 2006 e de 2010 para 2011, e pode-se analisar também que o comportamento da produção científica brasileira se manteve estável entre 2005 a 2008, e que após 2009 houve um aumento expressivo da quantidade de publicações, tanto no Brasil quanto no exterior, principalmente nos anos mais recentes, entre 2012 a 2014.

Identifica-se que houve crescimento consistente de aproximadamente $208 \%$ de 2005 para 2014 no total dos artigos brasileiros indexados, dessa forma, essa constatação evidencia certo ganho de forças da área pois vem aumentando sua frequência como tema em pesquisa científica. Uma das prováveis causas pode ser a crescente preocupação mundial quanto a melhoria dos aspectos ambientais, e por consequência, dos sociais, em consonância com a produção agrícola.

Pode-se constatar também que entre os anos de 2005 e 2014, houve nas publicações no Brasil, crescimento de aproximadamente $87 \%$ (de 23 em 2005 para 43 em 2014) da produção científica brasileira, com 324 pesquisas, porém com bastante oscilação.

0 aumento mais significativo ocorreu de 2008 a 2009 (52\%) e decréscimo mais relevante entre 2010 e 2011 (-23\%), com destaque para o ano de 2010 que teve a maior quantidade de artigos indexados dentro de todo o período analisado. Em síntese, as publicações brasileiras cresceram nos últimos dez anos, porém sem distribuição uniforme e com grandes irregularidades na comparação de um ano para outro. 
Em se tratando da produção brasileira publicada no exterior, identificou-se 346 trabalhos indexados e crescimento de aproximadamente 423\% (de 13 em 2005 para 68 em 2014), e evolução mais uniforme, com todos os anos tendo progresso em relação ao período anterior, situação que não ocorre somente quando se compara 2010 a 2011, com queda de $10 \%$ na quantidade de trabalhos indexados.

0 crescimento mais significativo ocorreu de 2008 a 2009 (72\%), já o ano de 2014 foi de maior quantidade de trabalhos indexados. Registrou-se também um aumento linear no decorrer dos três últimos anos pesquisados. A frequência de artigos brasileiros publicados no exterior apresentou crescimento de forma mais regular do que os publicados nacionalmente.

A evolução da produção científica ao longo do tempo demonstrou o fortalecimento e/ou o aumento do interesse por um determinado campo do conhecimento pela comunidade científica, o que nos remete a pensar que ainda não está consolidado (SAES, 2000).

Com isso, pode-se afirmar que os estudos dos indicadores socioambientais no agronegócio estão em constante atração de novos pesquisadores e conquistando maior visibilidade no decorrer dos dez últimos anos avaliados.

No que se trata da distribuição da produção científica brasileira por países, regiões, estados e cidades, a discussão se inicia com a apresentação dos países que publicaram os 346 artigos brasileiros (Tabela 2).

Tabela 2: Principais países de publicação dos artigos brasileiros sobre indicadores socioambientais no agronegócio na base Cab Abstracts, 2005-2014.

\begin{tabular}{c|ccccccccccc|c|c}
\hline País/Ano & 2005 & 2006 & 2007 & 2008 & 2009 & 2010 & 2011 & 2012 & 2013 & 2014 & $\begin{array}{c}\text { Total das } \\
\text { Publicações }\end{array}$ & $\begin{array}{c}\% \text { Sobre } 0 \\
\text { Total }\end{array}$ \\
\hline Reino Unido & 3 & 4 & 5 & 6 & 2 & 9 & 13 & 19 & 18 & 25 & 104 & 30,1 \\
\hline EUA & 2 & 2 & 2 & 1 & 8 & 4 & 4 & 10 & 11 & 9 & 53 & 15,3 \\
\hline Holanda & 1 & 2 & 3 & 6 & 8 & 1 & 4 & 6 & 6 & 13 & 50 & 14,5 \\
\hline França & 4 & - & 1 & 1 & 2 & 8 & 1 & 1 & 1 & 1 & 20 & 5,8 \\
\hline Alemanha & - & 1 & - & 3 & 2 & 2 & - & 1 & 3 & 4 & 16 & 4,6 \\
\hline Itália & - & 1 & 3 & - & 1 & 1 & 2 & - & 1 & 2 & 11 & 3,2 \\
\hline Suíça & - & - & - & - & 1 & 1 & - & 1 & 3 & 5 & 11 & 3,2 \\
\hline Espanha & - & 1 & 1 & - & 1 & 1 & - & 3 & - & 1 & 8 & 2,3 \\
\hline Quênia & - & - & - & - & - & - & 1 & - & 2 & 4 & 7 & 2,0 \\
\hline Outros & 3 & 2 & 2 & 1 & 6 & 14 & 12 & 11 & 11 & 4 & 66 & 19,1 \\
\hline Total Geral & 13 & 13 & 17 & 18 & 31 & 41 & 37 & 52 & 56 & 68 & 346 & 100,0 \\
\hline
\end{tabular}

Fonte: dados do autor a partir da pesquisa na Cab Abstracts (2015).

Analisando os países que publicaram os 346 artigos brasileiros, os três primeiros representam cerca de $60 \%$ do total das publicações, e levando em consideração que, de acordo com a Organização das Nações Unidas (ONU), o mundo possui 192 países, pode-se considerar que a produção científica brasileira sobre indicadores socioambientais no agronegócio foi publicada em aproximadamente $20 \%$ das nações ao redor do globo.

Quanto a análise dos artigos publicados no Brasil, os dados estão apresentados na Tabela 3, que apresenta a distribuição das publicações por região, Estados e cidades de maiores destaques, considerando a quantidade mínima de publicação de 6 artigos por Estado. 
Tabela 3: Distribuição das publicações no Brasil por região, Estado e cidade, sobre indicadores socioambientais no agronegócio na base Cab Abstracts, 2005-2014.

\begin{tabular}{|c|c|c|c|c|c|c|c|c|}
\hline Região & $\begin{array}{l}\text { Quantidade de } \\
\text { Publicações por } \\
\text { Região }\end{array}$ & \% por Região & $\begin{array}{c}\text { Estado em Destaque } \\
\text { na Região }\end{array}$ & $\begin{array}{l}\text { Quantidade de } \\
\text { Publicações por } \\
\text { Estado }\end{array}$ & $\begin{array}{l}\% \text { do Estado } \\
\text { sobre o Total }\end{array}$ & $\begin{array}{c}\text { Cidade em Destaque } \\
\text { por Estado }\end{array}$ & $\begin{array}{l}\text { Quantidade de } \\
\text { Publicações por } \\
\text { Cidade }\end{array}$ & $\begin{array}{l}\text { \% da Cidade } \\
\text { sobre o Total }\end{array}$ \\
\hline \multirow{5}{*}{ Sudeste } & \multirow{5}{*}{137} & \multirow{5}{*}{42,3} & \multirow{3}{*}{ Minas Gerais } & \multirow{3}{*}{66} & \multirow{3}{*}{20,4} & Viçosa & 25 & 7,7 \\
\hline & & & & & & Belo Horizonte & 20 & 6,2 \\
\hline & & & & & & Lavras & 10 & 3,1 \\
\hline & & & São Paulo & 49 & 15,1 & São Paulo & 21 & 6,5 \\
\hline & & & Rio de Janeiro & 21 & 6,5 & Rio de Janeiro & 20 & 6,2 \\
\hline \multirow{3}{*}{ Sul } & \multirow{3}{*}{75} & \multirow{3}{*}{23,1} & \multirow{2}{*}{ Paraná } & \multirow{2}{*}{47} & \multirow{2}{*}{14,5} & Londrina & 19 & 5,9 \\
\hline & & & & & & Curitiba & 13 & 4,0 \\
\hline & & & Rio Grande do Sul & 26 & 8,0 & - & - & - \\
\hline \multirow{3}{*}{ Centro Oeste } & \multirow{3}{*}{71} & \multirow{3}{*}{21,9} & \multirow{2}{*}{ Distrito Federal } & \multirow{2}{*}{60} & \multirow{2}{*}{18,5} & Brasilia & 48 & 14,8 \\
\hline & & & & & & Planaltina & 12 & 3,7 \\
\hline & & & Goiás & 10 & 3,1 & - & - & - \\
\hline \multirow{3}{*}{ Nordeste } & \multirow{3}{*}{38} & \multirow{3}{*}{11,7} & Ceará & 14 & 4,3 & Limoeiro do Norte & 11 & 3,4 \\
\hline & & & Rio Grande do Norte & 7 & 2,2 & - & - & - \\
\hline & & & Pernambuco & 6 & 1,9 & - & - & - \\
\hline Norte & 3 & 0,9 & - & - & - & - & - & - \\
\hline Total Geral & 324 & 100,0 & Total Geral & 306 & 94,4 & Total Geral & 199 & 61,4 \\
\hline
\end{tabular}

Fonte: dados do autor a partir da pesquisa na Cab Abstracts (2015).

A região brasileira de maior expressividade na publicação dos 324 artigos analisados foi a Sudeste com $42,3 \%$ das pesquisas, resultado que vai ao encontro do trabalho de Faria et al. (2011), que evidencia que em todas as áreas do conhecimento, a região citada possui liderança quanto à quantidade de publicações.

Tal situação pode ser associada à infraestrutura de pesquisa e desenvolvimento, a maior concentração de pesquisadores e recursos humanos especializados e ao maior volume de investimentos.

A localização geográfica dos fluxos de conhecimento também está estreitamente ligada ao desenvolvimento regional, na medida em que o aumento da capacidade de acesso de empresas que investem em Pesquisa e Desenvolvimento (P\&D) às fontes de conhecimento, pode ter seu efeito potencializado em termos de atividades inovativas caso essas fontes estejam integradas às redes de produção compartilhada de conhecimento técnico-científico (SIDONE et al., 2016).

Porém, como a região sudeste já se encontra com número elevado de publicações, o percentual de crescimento se tornou expressivo, mas inferior ao das demais regiões.

Essa diferenciação entre o Sudeste e as demais regiões, pelo menos em parte, refletiu os resultados das políticas de ciência e tecnologia implementadas pelos governos Federal e locais. Contudo, estão sendo elaboradas ações voltadas para a desconcentração da atividade científica e tecnológica e expansão para outras regiões do país. Mesmo assim a região Sudeste abrangeu a maior parte dos grupos de pesquisa em 2006, e desses, mais da metade dos pesquisadores tinham doutorado, além de receber a maior parte dos recursos do Conselho Nacional de Desenvolvimento Científico e Tecnológico (CNPq) (FARIA et al., 2011).

Vale destacar o desempenho da região Centro-Oeste na terceira posição com cerca de $22 \%$ das publicações, em comparação com os resultados de Faria et al. (2011), a situação apresentada diferiu quando se comparada à produção nacional em todas as áreas do conhecimento, pois o estado brasileiro com maior número de publicação foi São Paulo, seguido por Rio de Janeiro, Minas Gerais, Rio Grande do Sul e Paraná.

Quanto a identificação dos maiores estados publicadores brasileiros, o destaque ficou para Minas Gerais, Distrito Federal, São Paulo e Paraná, que agrupadas representaram $68,5 \%$ de toda a publicação nacional.

A geografia da produção e colaboração científica no país é marcada por intensa heterogeneidade espacial, com concentração sistemática da produção e dos fluxos de conhecimento nas regiões Sudeste e Sul, com destaque aos Estados que sediam 
universidades públicas (federais e estaduais) consolidadas no cenário acadêmico nacional, porém com padrões espaciais peculiares a cada domínio científico (SIDONE et al., 2016).

O Acre, Amapá, Rondônia, Roraima e Tocantins (região Norte), Alagoas e Maranhão (região Nordeste), e Mato Grosso (região Centro Oeste) não tiveram nenhum trabalho indexado com a temática de indicadores socioambientais no agronegócio na Cab Abstracts no período analisado, expondo de maneira mais clara a fragilidade da região Norte, bem como o Estado de Mato Grosso, no que se refere às publicações científicas.

Registrou-se que 49 cidades brasileiras publicaram artigos elaborados com a temática abordada, com destaque para as seis principais (Brasília, Viçosa, São Paulo, Belo Horizonte, Rio de Janeiro e Londrina) que representam $47 \%$ da publicação nacional. Neste tópico cabe ressaltar a importância da cidade de Brasília, que teve a maior frequência de publicações no período, situação que contribui de maneira considerável para que o CentroOeste ocupasse a terceira posição em termos de região.

Foram identificadas 108 cidades estrangeiras que constam publicações brasileiras a respeito do tema, sendo que as 12 principais cidades concentram 54,6\% dos artigos, com destaque para cidades localizadas no Reino Unido e Holanda, que ocupam as cinco primeiras posições (Tabela 4).

Tabela 4: Principais cidades de publicações no exterior sobre indicadores socioambientais no agronegócio na base Cab Abstracts, 2005-2014.

\begin{tabular}{|c|c|c|c|}
\hline País & $\begin{array}{c}\text { Cidade em Destaque } \\
\text { por País }\end{array}$ & $\begin{array}{c}\text { Quantidade de } \\
\text { Publicações por Cidade }\end{array}$ & $\begin{array}{l}\% \text { da Cidade } \\
\text { Sobre o Total }\end{array}$ \\
\hline \multirow{4}{*}{ Reino Unido } & Oxford & 62 & 17,9 \\
\hline & Londres & 15 & 4,3 \\
\hline & Abingdon & 10 & 2,9 \\
\hline & Chichester & 9 & 2,6 \\
\hline \multirow{3}{*}{ Holanda } & Amsterdam & 24 & 6,9 \\
\hline & Dordrecht & 15 & 4,3 \\
\hline & Wageningen & 8 & 2,3 \\
\hline França & Paris & 10 & 2,9 \\
\hline Estados Unidos & Filadélfia & 10 & 2,9 \\
\hline Itália & Roma & 10 & 2,9 \\
\hline Suíça & Genebra & 9 & 2,6 \\
\hline Quênia & Nairóbi & 7 & 2,0 \\
\hline- & Outras & 157 & 45,4 \\
\hline \multicolumn{2}{|c|}{ Total Geral } & 346 & 100,0 \\
\hline
\end{tabular}

Fonte: dados do autor a partir da pesquisa na Cab Abstracts (2015).

A produção brasileira publicada no exterior está concentrada em algumas cidades localizadas nos países citados, fato que não ocorre por exemplo com os Estados Unidos, que possui 53 trabalhos, mas estes bem pulverizados em diversas cidades.

Zago (2011), analisando a produção científica mundial e nacional de 1996 a 2008, identificou que a pesquisa brasileira teve um progresso quantitativo significativo no período, porém o progresso qualitativo foi menos expressivo e com impacto reduzido globalmente.

A situação descrita por Zago (2011) condiz em partes com o apresentado para o tema "indicadores socioambientais no agronegócio", pois o progresso na quantidade de pesquisas nos últimos dez anos é visível, mostrando a crescente preocupação com a temática. No entanto, pode-se analisar as contribuições efetivas que esse aumento de produção está causando, pois o progresso expressivo de quantidade e qualidade exigirá a adoção de novos padrões de organização e de gestão da produção científica e tecnológica nacional.

Quanto a investigação dos autores da produção científica que tiveram artigos publicados tanto no Brasil quanto exterior, foram identificados 2.224 autores (Tabela 5). 
Tabela 5: Distribuição da frequência de publicações por autor sobre indicadores socioambientais no agronegócio na base Cab Abstracts, 2005-2014.

\begin{tabular}{c|c|c|c}
\hline $\begin{array}{c}\text { Quantidade } \\
\text { de Autores }\end{array}$ & $\begin{array}{c}\text { Frequência de } \\
\text { Publicações por Autor }\end{array}$ & $\begin{array}{c}\text { Quantidade Total } \\
\text { de Publicações }\end{array}$ & $\begin{array}{c}\text { \% Sobre o Total } \\
\text { de Pulicações }\end{array}$ \\
\hline 2004 & 1 & 2004 & 80,0 \\
178 & 2 & 356 & 14,2 \\
30 & 3 & 90 & 3,6 \\
8 & 4 & 32 & 1,3 \\
2 & 5 & 10 & 0,4 \\
1 & 6 & 6 & 0,2 \\
1 & 8 & 8 & 0,3 \\
\hline \multicolumn{4}{r|}{} \\
\hline
\end{tabular}

Fonte: dados do autor a partir da pesquisa na Cab Abstracts (2015).

Esta situação expõe que a área pesquisada possui dispersão de autores, pois a maior parte deles possui poucas publicações, enquanto que os que lideram não conseguem, em comparação com o total de pesquisas, ter quantidade tão elevada de trabalhos publicados.

Em termos gerais, os resultados apresentaram poucos autores se dedicando continuamente à pesquisa e publicando sobre o tema, tendo em vista que somente doze deles tiveram quatro ou mais publicações, sendo: Cunha, M. H. com 8 trabalhos; Tourrand, J. F. com 6 pesquisas; Cerri, C. E. P. e Poccard-Chapuis, R. com 5 estudos; e Bursztyn, M., Cerri, C. C., Fernandes, M. F., Goncalves Junior, A. C., Lacerda, M. P. C., Rodrigues Filho, S., Scopel, E. e Xavier, J. H. V. com 4 publicações cada.

Em se tratando dos autores com maior frequência de artigos publicados, cabe destacar os quatro principais, os quais possuem as seguintes características:

a) Mariangela Hungria da Cunha - pesquisadora brasileira que trabalha na Empresa Brasileira de Pesquisa Agropecuária (Embrapa) Soja em Londrina, no Paraná;

b) Jean François Tourrand e René Jan Marie Poccard Chapuis - ambos pesquisadores da França que trabalham no Centro de Cooperação Internacional em Pesquisa Agronômica para o Desenvolvimento (CIRAD);

c) Carlos Eduardo Pellegrino Cerri - pesquisador brasileiro que atua na Escola Superior de Agricultura Luiz de Queiroz (ESALQ) da Universidade de São Paulo (USP).

Notou-se que entre os melhores classificados em termos de produção, tem-se grande contribuição dos autores franceses que já atuaram profissionalmente no Brasil, além dos brasileiros que trabalham para instituições consideradas renomadas na pesquisa nacional.

A situação revelada coincidiu com o trabalho de Lima et al. (2014), que analisaram a colaboração entre Brasil e França na pesquisa em Ciências Agrárias entre 2004 e 2013, no qual foi relatado que as coautorias entre Brasil e França estão em segundo lugar nas colaborações internacionais do Instituto Nacional de Pesquisa Agronômica da França, com $12,6 \%$ do total de artigos publicados, atrás apenas dos Estados Unidos com 37,7\%.

Além disso, constatou-se que a colaboração Brasil-França evoluiu entre 2004 e 2013 com uma média de 148,10 publicações por ano, a uma taxa de crescimento anual médio de $11,79 \%$, com destaque para os anos de 2006 (13,13\%), 2008 (22,22\%), 2009 e 2010 $(16,67 \%$ e $16,30 \%$ respectivamente).

Quando se trata das publicações nacionais, identificou-se 1.088 autores no geral, destes 1.002 possuem apenas 1 trabalho publicado, 79 com 2 pesquisas, e os mais expressivos (a, b) possuem 4 trabalhos, e os demais com 3 publicações (c, d, e, f), com as seguintes informações:

a) Affonso Celso Gonçalves Júnior - Doutor na Universidade Estadual do Oeste do Paraná, Campus de Marechal Cândido Rondon, Centro de Ciências Agrárias, no Paraná;

b) Marilusa Pinto Coelho Lacerda - Doutora na Universidade de Brasília, Faculdade de Agronomia e Medicina Veterinária, no Distrito Federal;

c) Paulo César de Lima e Waldênia de Melo Moura - ambos Doutores atuantes na Empresa de Pesquisa Agropecuária de Minas Gerais (EPAMIG), Secretaria de Agricultura e 
Abastecimento de Minas Gerais, Centro Tecnológico da Zona da Mata (CTZM), no estado mineiro;

d) Patrício Borges Maracajá - Doutor na Universidade Federal de Campina Grande, CCTA - Centro de Ciências e Tecnologias Agro alimentaria, na Paraíba;

e) Antônio Cordeiro de Santana - Doutor na Universidade Federal Rural da Amazônia, Instituto Socioambiental e dos Recursos Hídricos, no Pará;

f) Lourival Vilela - Mestre na Empresa Brasileira de Pesquisa Agropecuária, Centro de Pesquisa Agropecuária dos Cerrados, Embrapa Cerrados.

Conforme informações dos principais autores, observou-se que a maior parte deles está vinculado a instituições do Distrito Federal e Minas Gerais, com predominância da EPAMIG, não sendo essa uma das instituições que mais publicam no Brasil, mas possui dois autores no ranking de brasileiros com maior número de publicações indexadas, o que pode indicar que nela haja competências estabelecidas em indicadores socioambientais no agronegócio.

No que se refere aos autores com publicações brasileiras no exterior, os destaques ficaram por conta de Mariangela Hungria da Cunha com 6 trabalhos, Carlos Eduardo Pellegrino Cerri e Jean François Tourrand com 4 pesquisas.

Além desses, identificou-se mais dois autores com 4 publicações: Marcel Bursztyn e Saulo Rodrigues Filho, ambos da Universidade de Brasília, que é a instituição dominante entre os autores com mais trabalhos indexados na Cab Abstracts.

Pode-se afirmar que os dois autores brasileiros que mais se destacam em quantidade geral de artigos indexados sobre o tema, não os divulgam em sua maior parte no Brasil, sendo que os artigos da Mariangela Hungria da Cunha foram publicadas na Holanda (5) e no Reino Unido (1), e os de Carlos Eduardo Pellegrino Cerri no Reino Unido (3) e na Itália (1).

Quanto a abordagem das instituições de ensino que se destacaram em quantidade de publicações sobre o tema estudado, é visto que esta análise serviu para demonstrar tendência de engajamento ou entrada de instituições com publicação nas áreas em questão.

Na Cab Abstracts o campo instituição do autor é subestimado, pois a base registra apenas a instituição do primeiro autor da publicação. Com isso, a produção científica de uma instituição pode não ser contabilizada integralmente na base, pois dada esta limitação, acaba-se recuperando apenas as publicações onde os autores de tal instituição assinaram como primeiro autor, ignorando aquelas nas quais assinaram em coautoria.

Além do entrave citado, é importante destacar que no resultado da busca feita as informações trazidas no campo de instituição publicadora estrangeira em muitos casos continha o nome da editora (por exemplo as editoras comerciais Elsevier, Blackwell, entre outras) e não as instituições de afiliação dos autores, impossibilitando uma análise fidedigna deste item. Porém no caso dos trabalhos brasileiros, podem ser observadas as principais instituições na Tabela 6.

Tabela 6: Distribuição dos artigos brasileiros, por instituições de publicação, em indicadores socioambientais no agronegócio na base Cab Abstracts, 2005-2014.

\begin{tabular}{l|c|c|c}
\multicolumn{1}{c|}{ Instituição de Publicação } & Estado & $\begin{array}{c}\text { Quantidade } \\
\text { de Publicações }\end{array}$ & $\begin{array}{c}\text { \% Sobre } \\
\text { o Total }\end{array}$ \\
\hline Empresa Brasileira de Pesquisa Agropecuária - Embrapa & Diversos & 58 & 17,9 \\
Empresa de Pesquisa Agropecuária de Minas Gerais (EPAMIG) & Minas Gerais & 20 & 6,2 \\
Sociedade Brasileira de Economia e Sociologia Rural (SOBER) & Distrito Federal & 18 & 5,6 \\
Ministério da Agricultura, Pecuária e Abastecimento (MAPA) & Distrito Federal & 13 & 11 \\
Centro Federal de Educação Tecnológica (CEFET) Limoeiro-CE & Ceará & 1,0 & 3,4 \\
Universidade Estadual do Oeste do Paraná (UNIOESTE) & Paraná & 11 & 3,4 \\
Instituto de Economia Agrícola (IEA) & São Paulo & 10 & 3,1 \\
Sociedade Brasileira de Ciência do Solo (SBCS) & Minas Gerais & 9 & 2,8 \\
Universidade Federal do Paraná (UFPR) & Paraná & 9 & 2,8 \\
Universidade Federal de Santa Maria (UFSM) & Rio Grande do Sul & 8 & 2,5 \\
Universidade Federal de Lavras (UFLA) & Minas Gerais & 8 & 2,5 \\
Outras & - & 149 & 46,0 \\
\hline \multicolumn{1}{c|}{ Total Geral } & $\mathbf{3 2 4}$ & $\mathbf{1 0 0 , 0}$ \\
\hline
\end{tabular}

Fonte: dados do autor a partir da pesquisa na Cab Abstracts (2015). 
Com base nas informações acima, o destaque fica por conta da Embrapa, que possui vínculo com o Ministério da Agricultura, Pecuária e Abastecimento, indicando que o país considera os indicadores socioambientais no agronegócio um ramo de estudo de importância, pois até mesmo órgãos governamentais, ou vinculados, editam revistas que publicam parte considerável dos trabalhos da área.

Em termos gerais, foram identificadas 86 instituições responsáveis por revistas brasileiras que publicaram artigos indexados sobre o tema, as mais bem colocadas representadas na Tabela 7 concentraram $54 \%$ da produção científica brasileira. Com exceção da Embrapa, que possui centros de pesquisa instalados em quase todo o território nacional, as primeiras posições do ranking foram ocupadas por instituições das regiões Sul, Sudeste e Centro-Oeste do país, com destaque para os estados de Minas Gerais, com 37 artigos, Distrito Federal, com 31, e Paraná, com 20.

Outro fator de destaque ficou por conta das publicações em revista do Centro Federal de Educação Tecnológica de Limoeiro do Norte no Ceará, que se encontra entre as primeiras colocadas.

Vale ressaltar que a USP, que tem predominância na produção científica nacional, nos resultados de busca sobre indicadores socioambientais no agronegócio aparece com baixa frequência de publicações de artigos sobre o tema, registrando apenas dois artigos, tendo a sua frente instituições de menor expressão e conhecimento no que se refere a produção científica nacional.

Quanto aos periódicos, a produção científica brasileira foi publicada em 367 diferentes revistas científicas, no entanto observou-se que houve maior concentração de artigos em revistas nacionais.

Foram usados 122 periódicos para publicação dos 324 artigos, enquanto que no exterior o uso foi de 245 periódicos para os 346 artigos, revelando que no exterior as publicações foram mais fragmentadas, e no Brasil o uso de algumas revistas absorveram quantidade expressiva de trabalhos.

Fato que comprova o descrito é que os 20 primeiros periódicos no ranking nacional publicaram 51,2\% dos trabalhos indexados na Cab Abstracts. Ao passo que no exterior os 20 primeiros periódicos concentraram $22 \%$ dos artigos indexados na mesma base.

Os periódicos que apresentaram maior frequência de publicações no Brasil foram o Informe Agropecuário, a Revista de Economia e Sociologia Rural, os Cadernos de Ciência \& Tecnologia e a Revista de Política Agrícola. A Tabela 7 apresenta as revistas melhores ranqueadas. 
Tabela 7: Distribuição de artigos brasileiros, por periódicos científicos no Brasil e no exterior, em indicadores socioambientais no agronegócio na base Cab Abstracts, 2005-2014.

\begin{tabular}{|c|c|c|c|c|c|c|c|c|c|c|c|c|c|c|c|}
\hline Periódico & Referência & $\begin{array}{r}\text { Estrato } \\
\text { Capes } \\
\end{array}$ & $\begin{array}{l}\text { Área de } \\
\text { Avaliação }\end{array}$ & 2005 & 2006 & 2007 & 2008 & 2009 & 2010 & 2011 & 2012 & 2013 & 2014 & Total & $\begin{array}{l}\text { \% Sobre } \\
\text { o Total }\end{array}$ \\
\hline Informe Agropecuário & Brasil & B4 & Interdisciplinar & - & - & 1 & 3 & 6 & 4 & - & 1 & - & 4 & 19 & 5,9 \\
\hline Revista de Economia e Sociologia Rural & Brasil & B1 & Interdisciplinar & 2 & 1 & 2 & 1 & - & 3 & 3 & 1 & 1 & 4 & 18 & 5,6 \\
\hline Cadernos de Ciência \& Tecnologia & Brasil & B3 & Interdisciplinar & 7 & - & - & - & - & - & - & 4 & - & 1 & 12 & 3,7 \\
\hline Revista de Política Agrícola & Brasil & B2 & Interdisciplinar & 1 & - & 1 & - & - & 1 & 2 & 1 & 5 & 1 & 12 & 3,7 \\
\hline $\begin{array}{l}\text { Revista Verde de Agroecologia e } \\
\text { Desenvolvimento Sustentavel }\end{array}$ & Brasil & B4 & Interdisciplinar & - & - & - & 1 & - & 1 & 3 & 3 & 2 & 1 & 11 & 3,4 \\
\hline Revista Brasileira de Ciência do Solo & Brasil & $\mathrm{A} 2$ & Interdisciplinar & - & - & 2 & 1 & - & 1 & 2 & 2 & 1 & - & 9 & 2,8 \\
\hline Ciência Rural & Brasil & $\mathrm{A} 2$ & Interdisciplinar & - & 2 & 1 & - & 2 & 1 & 1 & - & - & 1 & 8 & 2,5 \\
\hline Informações Econômicas & Brasil & B3 & Interdisciplinar & 2 & 1 & - & - & 2 & - & - & 2 & - & 1 & 8 & 2,5 \\
\hline Organizações Rurais e Agroindustriais & Brasil & B2 & Interdisciplinar & - & - & - & - & - & - & 1 & 2 & 3 & 2 & 8 & 2,5 \\
\hline Ciência \& Saúde Coletiva & Brasil & B1 & Interdisciplinar & - & - & 1 & - & 2 & - & - & - & 1 & 3 & 7 & 2,2 \\
\hline Documentos - Embrapa Soja & Brasil & B5 & Ciências Agrárias & - & - & 1 & 2 & 2 & - & - & - & 1 & 1 & 7 & 2,2 \\
\hline Scientia Agraria Paranaensis & Brasil & B5 & Interdisciplinar & - & 1 & - & - & - & 2 & 2 & - & 2 & - & 7 & 2,2 \\
\hline Outros & Brasil & - & - & 11 & 13 & 17 & 15 & 21 & 31 & 20 & 23 & 23 & 24 & 198 & 61,1 \\
\hline \multicolumn{4}{|c|}{ Total Geral } & 23 & 18 & 26 & 23 & 35 & 44 & 34 & 39 & 39 & 43 & 324 & 100,0 \\
\hline Soil \& Tillage Research & Exterior & $\mathrm{A} 2$ & Interdisciplinar & - & - & - & - & - & 1 & 1 & 1 & 1 & 3 & 7 & 2,0 \\
\hline $\begin{array}{c}\text { Innovation and Sustainable } \\
\text { Development in Agriculture and Food }\end{array}$ & Exterior & $\mathrm{A} 2$ & - & - & - & - & - & - & 6 & - & - & - & - & 6 & 1,7 \\
\hline $\begin{array}{c}\text { Communications in Soil Science and } \\
\text { Plant Analysis }\end{array}$ & Exterior & B1 & Interdisciplinar & - & - & - & - & 2 & 1 & - & 1 & 1 & - & 5 & 1,4 \\
\hline Land Use Policy & Exterior & A1 & Interdisciplinar & - & - & - & - & - & - & - & - & 1 & 4 & 5 & 1,4 \\
\hline Science of the Total Environment & Exterior & A1 & Interdisciplinar & - & 1 & - & - & - & 1 & - & 1 & 1 & 1 & 5 & 1,4 \\
\hline African Journal of Agricultural Research & Exterior & B1 & Interdisciplinar & - & - & - & - & - & - & - & - & 1 & 3 & 4 & 1,2 \\
\hline Cahiers Agricultures & Exterior & B2 & Interdisciplinar & 3 & - & - & - & - & 1 & - & - & - & - & 4 & 1,2 \\
\hline Water, Air, and Soil Pollution & Exterior & B2 & Interdisciplinar & - & - & - & - & 1 & 1 & - & - & 1 & 1 & 4 & 1,2 \\
\hline Outros & Exterior & - & - & 10 & 12 & 17 & 18 & 28 & 30 & 36 & 49 & 50 & 56 & 306 & 88,4 \\
\hline \multicolumn{4}{|c|}{ Total } & 13 & 13 & 17 & 18 & 31 & 41 & 37 & 52 & 56 & 68 & 346 & 100,0 \\
\hline
\end{tabular}

Fonte: dados do autor a partir da pesquisa na Cab Abstracts e da Coordenação de Aperfeiçoamento de Pessoal de Nível Superior (Capes) (2015).

Entre os destaques citados em frequência de publicações, o periódico Informe Agropecuário, vinculado a Empresa de Pesquisa Agropecuária de Minas Gerais (EPAMIG), ocupa o primeiro lugar, em segundo encontra-se a Revista de Economia e Sociologia Rural, que visa divulgar e difundir os resultados de pesquisas nas áreas de economia, administração, extensão e sociologia rural, publicação trimestral da Sociedade Brasileira de Economia e Sociologia Rural (SOBER), instituição também já citada que ficou em terceira colocada no ranking.

O periódico Cadernos de Ciência \& Tecnologia, criado em 1984, com o título Cadernos de Difusão de Tecnologia, é uma publicação interdisciplinar quadrimestral, editada pela Embrapa, que tem como objetivo fomentar a reflexão e o debate com textos sobre as questões tecnológicas e socioeconômicas ligadas ao desenvolvimento rural. Em quarto colocada a Revista de Política Agrícola, publicação trimestral da Secretaria de Política Agrícola do Ministério da Agricultura, Pecuária e Abastecimento (MAPA).

Em comparação com o trabalho de Vargas (2014), o periódico com maior produção científica em Ciências Agrárias entre 2000 e 2011 foi a Revista Brasileira de Zootecnia, que na busca com o tema de indicadores socioambientais no agronegócio também apresentou resultados. Porém, não foram tão significativos para permear as primeiras posições, tendo publicado 4 artigos, comprovando que a área estudada neste trabalho não representa foco de pesquisas para a revista.

Com vistas a identificar a qualidade dos principais periódicos científicos que publicaram artigos sobre o tema, foi pesquisada a classificação do estrato de acordo com o Qualis da Capes, conforme apresentado na Tabela 7.

Neste quesito destaca-se a Revista Brasileira de Ciência do Solo (Qualis A2) que é um periódico bimestral de divulgação científica publicado pela Sociedade Brasileira de Ciência do Solo (SBCS), desde 1977, classificada mundialmente entre os melhores periódicos de Ciência do Solo não publicadas em inglês; e o periódico Ciência Rural (Qualis A2), existente 
desde 1971, quando foi criada com nome de Revista do Centro de Ciências Rurais, vinculada a Universidade Federal de Santa Maria (UFSM).

Ambos possuem estrato elevado na área de avaliação Interdisciplinar, o que as classificam com alto fator de impacto elevando a importância dos periódicos científicos em suas respectivas áreas.

Os periódicos com menor classificação Qualis foram: Documentos - Embrapa Soja e Scientia Agraria Paranaensis, tendo em vista que ambos encontram-se com estrato B5, o primeiro na área de avaliação de Ciências Agrárias e o segundo na área Interdisciplinar.

As informações relacionadas aos trabalhos publicados no exterior, tem-se como destaque na primeira posição a Soil \& Tillage Research, que dos 7 artigos indexados, 4 foram publicados no Reino Unido e 3 na Holanda, este um periódico internacional do grupo Elsevier, especializado em pesquisa e desenvolvimento no preparo do solo e sua relação com o uso da terra, a produção agrícola e o meio ambiente.

Em seguida vem o Innovation and Sustainable Development in Agriculture and Food, o qual não se trata de uma revista científica, mas sim de um simpósio que aconteceu em Monpellier na França, em 2010, que discorreu sobre a temática da inovação e desenvolvimento sustentável na agricultura e na alimentação.

Do terceiro ao quinto classificados, observou-se a mesma representatividade na publicação da produção científica, importante destacar que a Land Use Policy e a Science of the Total Environment também pertencem ao grupo Elsevier.

Em síntese, as publicações dos artigos brasileiros estão mais concentradas em alguns periódicos científicos que se relacionam diretamente às instituições que se destacaram em quantidade de artigos publicados, já as pesquisas publicadas no exterior não se apresentam concentradas em poucos periódicos.

\section{CONSIDERAÇÕES FINAIS}

Ao analisar a parcela da literatura sobre os Indicadores Socioambientais no Agronegócio, a motivação foi de diagnosticar se as produções científicas dos últimos anos estão focadas em versar sobre a evolução da produção agrícola, aliando a isso a preocupação que se deve ter com o impacto ambiental e social causado por tal prática.

De forma ampla, pôde-se constatar que a preocupação das produções científicas nacionais em se escrever sobre os temas estudados vem crescendo com o passar do tempo, percebe-se que há uma enorme diversidade de autores, e destaque para regiões e Estados amplamente desenvolvidos em termos de infraestrutura e universidades, porém o Mato Grosso, responsável por grande parte do agronegócio nacional, que deveria focar suas pesquisas na relação desse segmento da economia com o desenvolvimento ambiental e social, não possui impacto nenhum em termos de produção científica nessas temáticas.

Com o intuito de especificar de maneira mais detalhada a abordagem global citada e confrontar o objetivo da pesquisa, que foi de avaliar a expressão científica de Mato Grosso na pesquisa brasileira sobre indicadores socioambientais no agronegócio na base de dados internacional Cab Abstracts, com os resultados encontrados, a seguir estão caracterizadas as principais informações geradas pelo estudo.

Constatou-se tendência de crescimento nas publicações nos últimos dez anos, tanto em âmbito nacional como no exterior, havendo, a partir de 2011, preferência pelos periódicos internacionais em termos de quantidade de artigos publicados.

Com relação às pesquisas publicadas nacionalmente, coube destaque para as regiões Sudeste e Sul, e em termos de estados os principais foram Minas Gerais, Distrito Federal, São Paulo e Paraná, e no comparativo das cidades Brasília, Viçosa, São Paulo, Rio de Janeiro e Belo Horizonte ficaram mais bem ranqueadas.

Já as publicações internacionais se concentraram mais no Reino Unido, Estados Unidos, Holanda e França, com evidência para as cidades de Oxford, Londres, Amsterdam e Dordrecht.

Referindo-se aos autores, os que se destacaram em termos de quantidade de artigos publicados indexados na Cab Abstracts, foram dois brasileiros: Mariangela Hungria da Cunha e Carlos Cerri, e dois franceses: Jean François Tourrand e René Jan Marie Poccard Chapuis. 
Para a análise das instituições nacionais publicadoras as primeiras posições ficaram para a Embrapa, a EPAMIG e a SOBER, sendo os periódicos Informe Agropecuário e Revistas de Economia e Sociologia Rural os que mais publicaram artigos com a temática.

Referente a expressão científica do Estado de Mato Grosso na pesquisa brasileira, identificou-se que o mesmo não possui nenhuma produção publicada, este resultado apresenta cenário contraditório, visto que se trata do Estado de maior expressão no agronegócio no país, porém sem visibilidade científica sobre a temática.

Diante disso, fica evidente a necessidade de Mato Grosso fazer maior investimento em ciência e tecnologia, voltado para pesquisas sobre o tema, a serem realizadas por grupos vinculados às Instituições públicas e privadas do Estado, com a finalidade de se obter crescimento em termos de publicações científicas em periódicos que possuem representatividade nos temas. Por meio do descrito analisa-se que o objetivo da pesquisa foi atingido.

Vale ressaltar que, no que se refere ao setor público, a correta compreensão da importância da participação acadêmica pode ajudar o processo de formulação de políticas relacionadas a Ciência e Tecnologia no Brasil, tendo como finalidade a melhoria da produção em regiões ainda insipientes, não somente em termos de quantidade, mas também na qualidade desses trabalhos.

Além disso, no que tange ao setor privado, faz-se necessário compreender que o desenvolvimento regional se dá em locais onde há um fluxo de conhecimento avançado, com isso, na medida em que há aumento de empresas que investem em pesquisa e desenvolvimento e que conseguem acessar essas fontes de conhecimento, pode gerar um efeito potencial favorável a melhoria da produção científica.

\section{REFERÊNCIAS}

ANDRADE, F. S. Análise bibliométrica da produção científica de pesquisadores e referências de um periódico da engenharia de produção. 2012. Dissertação (Mestrado em Engenharia de Produção)-Universidade Federal do Rio Grande do Sul, Porto Alegre, 2012. Disponível em: <https://www.lume.ufrgs.br/bitstream/handle/10183/61399/000864293.pdf?sequence=1>. Acesso em: 20 out. 2014

ASSAD, E. D., MARTINS, S. C., PINTO, H. S. Sustentabilidade no Agronegócio Brasileiro. In: Coleção de estudos sobre diretrizes para uma economia verde no Brasil. 2012. Disponível em: <http://fbds.org.br/fbds/IMG/pdf/doc-553.pdf>. Acesso em: 14 dez. 2014.

FARIA, L. I. L. et al. Análise da produção científica a partir de publicações em periódicos especializados. In:__ Indicadores de Ciência, Tecnologia e Inovação em São Paulo. São Paulo: Fapesp, 2010. Disponível em: <http://www.fapesp.br/indicadores/2010/volume1/cap4.pdf>. Aceso em: 24 jul. 2015.

GAMBA, C. Avaliação da vulnerabilidade socioambiental dos distritos do município de São Paulo ao processo de escorregamento. Dissertação (Mestrado em Geografia)-Faculdade de Filosofia, Letras e Ciências Humanas, Universidade de São Paulo, São Paulo, 2011. Disponível em: <http://www.teses.usp.br/teses/disponiveis/8/8136/tde-15122011-105031/pt-br.php>. Acesso em: 26 mai. 2015.

HENRIQUEZ, C. et. al. A utilização de indicadores socioambientais no processo de tomada de decisão para o desenvolvimento sustentável da microbacia do Rio Sagrado: relevância da interdisciplinaridade e transdisciplinaridade nas abordagens socioambientais. 2009. Disponível em: <http://hygeia.fsp.usp.br/siades/documentos/Publicacoes/Artigo_2f.pdf>. Acesso em: 27 mai. 2014.

JÚNIOR, C. M. et al. Estudo bibliométrico da sustentabilidade ambiental: os 15 anos do Simpósio de Administração da Produção, Logística e Operações Internacionais - SIMPOI. Journal of the Health Sciences Institute., v.31, n. 2, p. 123-31, 2013. Disponível em: <http://www.unip.br/comunicacao/publicacoes/ics/edicoes/2013/02_abrjun/V31_n2_2013_p123a131.pdf>. Acesso em: 01 out. 2014.

LIMA, R. A.; et al. A colaboração Brasil-França na pesquisa em ciências agrárias (2004-2013). In: Encontro Brasileiro de Bibliometria e Cientometria, 4., 2014, Recife. Disponível em: <http://prodinra.inra.fr/ft?id=58A6EFAC-0935-44AB-93F3-2F8926753BAF >. Acesso em: 11 ago. 2015 . 
LOUETTE, A. (org.). Indicadores de Nações: uma contribuição ao diálogo da sustentabilidade. São Paulo: WHH, 2009. Disponível em: <http://www.portalodm.com.br/publicacao/183/indicadores-denacoes---uma-contribuicao-ao-dialogo-da-sustentabilidade>. Acesso em: 26 mai. 2015.

MATO GROSSO: SEPLAN - SECRETARIA DE ESTADO DE PLANEJAMENTO E COORDENAÇÃO GERAL. In: . Plano Plurianual 2012-2015. 2011. Disponível em:

<http://www.seplan.mt.gov.br/arquivos/DOCUMENTO\%20FINAL\%20\%20122012\%20\%20PPA\%20\%202012\%20-\%202015.pdf>. Acesso em: 14 dez. 2014.

PUERTA, A. A. Pesquisa em nanotecnologia para o agronegócio: indicadores bibliométricos de produção científica entre 2001 a 2010. 2012. 156f. Dissertação (Mestrado em Ciência, Tecnologia e Sociedade)-Centro de Educação e Ciências Humanas, Universidade Federal de São Carlos, São Carlos, 2012. Disponível em:

<http://www.bdtd.ufscar.br/htdocs/tedeSimplificado//tde_busca/arquivo.php?codArquivo=5184>. Acesso em: 18 ago. 2014.

SAES, S. G. Estudo bibliométrico das publicações em economia da saúde, no Brasil, 1989-1998. 2000. 104f. Dissertação (Mestrado em Administração de Serviços de Saúde)-Faculdade de Saúde Pública, Universidade de São Paulo, São Paulo, 2000. Disponível em:

<http://www.teses.usp.br/teses/disponiveis/6/6135/tde-01032002-132854/pt-br.php>. Acesso em: 08 ago. 2015.

SCANDAR NETO, W. J., et al. Sistemas de Indicadores ou Indicadores Sintéticos: do que precisam os gestores de Programas Sociais? In: Encontro Nacional de Estudos Populacionais, 16., 2008, Caxambu. Disponível em:

<http://www.abep.nepo.unicamp.br/encontro2008/docsPDF/ABEP2008_1511.pdf>. Acesso em: 26 mai. 2015.

SIDONE, O. J. G., et al. A ciência nas regiões brasileiras: evolução da produção e das redes de colaboração científica. TransInformação, 15-31, jan./abr., 2016, Campinas. Disponível em: <http://www.scielo.br/pdf/tinf/v28n1/0103-3786-tinf-28-01-00015.pdf >. Acesso em: 19 nov. 2016.

SOUZA, M. T. S., et al. Perfil e evolução da pesquisa em Sustentabilidade Ambiental: uma Análise Bibliométrica. 2011. In: Encontro da ANPAD,35, 2011, Rio de Janeiro. Disponível em: <http://www.anpad.org.br/admin/pdf/GOL2087.pdf>. Acesso em: 01 out. 2014.

SOUZA, C. D., FARIA, L. I. L. Indicadores bibliométricos de produção científica: Contribuições para políticas públicas na Citricultura brasileira. In: Simpósio Nacional de Tecnologia e Sociedade, 4., 2013, Curitiba. Disponível em: <http://www.esocite.org.br/eventos/tecsoc2011/cdanais/arquivos/pdfs/artigos/gt023-indicadoresbibliometricos.pdf>. Acesso em: 01 out. 2014.

VARGAS, R. A. A Produção científica brasileira em ciências agrárias indexada na Web of Science: características e redes de colaboração (2000-2011). 2014. 131f. Dissertação (Mestrado em Comunicação e Informação)-Faculdade de Biblioteconomia e Comunicação, Universidade Federal do Rio Grande do Sul, Porto Alegre, 2014. Disponível em:

<https://www.lume.ufrgs.br/bitstream/handle/10183/102304/000934241.pdf?sequence=1>. Acesso em: 11 ago. 2015.

ZAGO, M. A. Perfil da produção científica brasileira. São Paulo: Fapesp, 2011. Disponível em: <http://www.fapesp.br/eventos/2011/06/Marco_Antonio.pdf>. Acesso em: 09 ago. 2015.

Editores do artigo: Adilson Luiz Pinto, Rafaela Paula Schmitz e Enrique Muriel-Torrado 